\title{
Protective effects and mechanisms of microRNA-182 on oxidative stress in RHiN
}

https://doi.org/10.1515/biol-2019-0045

Received December 23, 2018; accepted May 6, 2019

\begin{abstract}
To explore protective effects and related mechanisms of microRNA-182(miR-182) on oxidativestress in rat hippocampal neurons (RHiN), RHiN cells. As the results, the survival rate and superoxide dismutase levels in $\mathrm{H}_{2} \mathrm{O}_{2}$ group were significantly lower than $\mathrm{H}_{2} \mathrm{O}_{2}+$ miR-182 group (all $\mathrm{P}<0.05$ ). The malondialdehyde levels and apoptosis rate in $\mathrm{H}_{2} \mathrm{O}_{2}+$ miR-182 group were significantly lower than $\mathrm{H} 2 \mathrm{O} 2$ group (all $\mathrm{P}<0.05$ ). The mRNA levels and expression levels of mTOR and PI3K in $\mathrm{H}_{2} \mathrm{O}_{2}+$ miR-182 group were higher than those in $\mathrm{H}_{2} \mathrm{O}_{2}$ group (both $\mathrm{P}<0.05$ ). The experiment of cerebral ischemic oxidative stress model rats showed that the survival rate, apoptosis rate, malondialdehyde and superoxide dismutase levels in miR-182 group were better than model control group. The positive staining intensity of phosphoinositide 3-kinase (mTOR) and phosphoinositide 3-kinase (PI3K) in model control group were significantly lower than miR-182 group (all $\mathrm{P}<0.05$ ). Increased levels of miR-182 can reduce the damage of $\mathrm{H} 2 \mathrm{O} 2$ treatments in $\mathrm{RHiN}$ cells. Oxidative stress is decreased in the neuronal cells possibly by activation of the PI3K-AKT-mTOR pathway.
\end{abstract}

Keywords: miR-182, rat hippocampal neurons, oxidative stress

\section{Introduction}

Oxidative stress mainly refers to the large number of reactive oxygen species (ROS), reactive nitrogen species (RNS) and other free radicals of the oxidation system. These species are generated due to pathological

\footnotetext{
*Corresponding author: Lihua Li, Colleges of Medicine, Jishou University, Jishou, Hunan Province, P.R. China,

E-mail: 13262569@qq.com

Lihua Li, Wenna Peng, Department of Rehabilitation Medicine, The Second Xiangya Hospital of Central South University, Changsha, Hunan Province, P.R. China

Xiangrong Tian, Colleges of Biology and Environmental Sciences, Jishou University, Jishou, Hunan Province, P.R. China
}

stimulation and promote lipid peroxidation of the cell membrane system, leading to decline in DNA, protein, and other macromolecular activities [1]. The nerve tissue in the hippocampus is involved in memory and neural information processing. This part of the brain tissue is injured by oxidative stress, which may lead to neurological diseases in patients [2]. Studies have discovered that brain tissue of patients with vascular dementia and cerebral infarction exhibits cerebral ischemia, hypoxia and other inflammatory symptoms. The levels of ROS, nitric oxide synthase (NOS) and other free radicals in the brain tissue are increased as a result. The result of these processes is an aggravation of the patients' oxidative stress [3]. Gong et al. have found that free radicals in the brain tissue of patients with senile dementia are high, causing lipid peroxidation in hippocampal neurons leading to their dysfunction. Outcomes of such damage are manifest as a poorer quality of life and a decrease in mental processes [4].

microRNA-182 (miR-182) is a single-stranded noncoding RNA consisting of 23 nucleotides. It was initially discovered in murine retinal tissue, and was found to regulate retinal sensitization in mice [5]. Studies have shown that miR-182 has a wide range of applications in biological systems. Metabolic and cellular division functions are regulated by control of target gene expression levels, and are closely related to many diseases, including those associated with nerve injury, tumor development, and depression [6]. Kho et al. have found that miR-182 can reduce ROS levels of cardiomyocytes in rats as well as normalise the apoptosis rate of the cardiomyocytes [7]. At present, there are few studies which detail the antioxidative protective effects of miR-182 on hippocampal neurons. The aim of this study is to explore protective effects and related mechanisms of miR-182 on oxidative stress in rat hippocampal neurons (RHiN). 


\section{Materials and methods}

\subsection{Cell groupings and culture}

RHiN cells were purchased from Shanghai YS Industrial Co., Ltd., China. Dulbecco's Modified Eagle Medium (DMEM) culture media, consisting of $10 \%$ fetal bovine serum, penicillin $(150 \mathrm{U} / \mathrm{mL})$, and streptomycin (120 U/ $\mathrm{mL}$ ), were all purchased from Shanghai Beinuo Biological Technology Co., Ltd., China. Cell culture groups were defined by their treatments: RHiN in blank control group were routinely cultured with DMEM media culture. Hippocampal neurons in miR-182 group were transfected with miR-182 (Guangzhou RiboBio Co., Ltd., China) according to Lipofectamine 2000 Kit instructions (Beijing Solarbio Science \& Technology Co., Ltd., China). A total of $200 \mu \mathrm{mol} / \mathrm{L}$ of $\mathrm{H}_{2} \mathrm{O}_{2}$ solution (Sangon Biotech (Shanghai) Co., Ltd., China) was added to the culture medium of the hippocampal cells in $\mathrm{H}_{2} \mathrm{O}_{2}$ group. A total $200 \mu \mathrm{mol} / \mathrm{L}$ of $\mathrm{H}_{2} \mathrm{O}_{2}$ solution and $80 \mu \mathrm{mol} / \mathrm{L}$ of mTOR protein (Beijing Biolab Technology Co., Ltd., China) were added to the culture medium of the hippocampal cells in mTOR $+\mathrm{H}_{2} \mathrm{O}_{2}$ group. A total $8 \mu \mathrm{mol} / \mathrm{L}$ of rapamycin solution (Sigma Co., Ltd., Germany) was added to the culture medium of the hippocampal cells in mTOR inhibitor group. A total 10 $\mu \mathrm{mol} / \mathrm{L}$ of MHY1485 solution (MedChemExpress Co., Ltd., USA) was added to the culture medium of the hippocampal cells in mTOR activator group. Hippocampal neurons in $\mathrm{H}_{2} \mathrm{O}_{2}+$ miR-182 group were transfected with miR-182 and supplemented with $200 \mu \mathrm{mol} / \mathrm{L} \mathrm{H}_{2} \mathrm{O}_{2}$ solution. All cell cultures were placed in an incubator at $37^{\circ} \mathrm{C}$, with $5 \% \mathrm{CO}_{2}$.

\subsection{Detection of activity and apoptosis levels in RHiN cells}

Cultured cells at exponential growth phase were seeded into 96-well culture plates, $1 \times 10^{4}$ cells per well. Cells were cultured for $24 \mathrm{~h}$ according to treatment groups, washed 3 times with ice-cold phosphate buffer saline (PBS), followed by addition of $25 \mu \mathrm{L}$ of MTT $(5 \mathrm{~g} / \mathrm{L})$ (Beijing Biolab Technology Co., Ltd., China) to each well. Cells were cultured for an additional $4 \mathrm{~h}$. Following the incubation with MTT, the supernatant was discarded and $150 \mu \mathrm{L}$ dimethylsulfoxide (DMSO, Shanghai Yeasen Biotechnology Co., Ltd., China) was added to each well for dissolution. A micro oscillator was used for $10 \mathrm{~min}$ to dissolve completely any crystals formed during the incubation. The optical density (OD) at $492 \mathrm{~nm}$ was determined using microplate reader (Shanghai Flash Spectrum Biological Technology Co., Ltd., China). The method for calculating the cell survival rate is as follows: survival rate $=(O D$ in the treatment group / OD in the blank control group) x 100\%.

Flow cytometry was used to analyze the effect of siRNA transfection. RHiN treatment groups were cultured to logarithmic phase. These cells were digested for $5 \mathrm{~min}$ using $0.25 \%$ pancreatin (Shanghai Yeasen Biotechnology Co., Ltd., China), and then centrifuged for $10 \mathrm{~min}$ at 2,000 $\mathrm{rpm} / \mathrm{min}$. After collecting cells and washing twice with icecold PBS, $600 \mu \mathrm{L}$ ethanol was added to cells, and placed at $-4^{\circ} \mathrm{C}$ overnight. Cells were washed twice more with PBS and $350 \mu \mathrm{L}$ binding buffer was added to resuspend cells. Annexin V-Enhanced Green Fluorescent Protein (EGFP) (10 $\mu \mathrm{L}$, Shanghai Yubo Biological Technology Co., Ltd., China) was added for mixing. Propidium iodide (PI) (5 $\mu \mathrm{L}$ ) staining solution was added, incubated for $10 \mathrm{~min}$ at room temperature, and flow cytometry (Beckman Coulter Co., Ltd., USA) was carried out to detect cellular apoptosis levels. The excitation wavelength was set to $\mathrm{Ex}=488 \mathrm{~nm}$, and the emission wavelength was set to $E m=530 \mathrm{~nm}$.

\subsection{Detection of superoxide dismutase (SOD) and malondialdehyde (MDA) levels in RHiN cells}

Exponential phase cells were seeded into 96-well culture plates, $1 \times 10^{4}$ cells per well. Cells were cultured for $24 \mathrm{~h}$ according to treatment groups. Following cell collection, radioimmunoprecipitation assay (RIPA) buffer (Shanghai Yeasen Biotechnology Co., Ltd., China) was added and cells were incubated for $10 \mathrm{~min}$ at $4^{\circ} \mathrm{C}$, then centrifuged for $5 \mathrm{~min}$ at 12,000 rpm. Supernate was taken in lowtemperature preservation. The SOD levels of RHiN were detected by enzyme-linked immuno sorbent assay (ELISA) according to the manufacturer's instructions (Shanghai Jianglai Biotech Co., Ltd., China). The OD value of each well was determined at $450 \mathrm{~nm}$. Thiobarbituric acid (TBA) was utilized to detect the levels of MDA, according to the MDA Assay Kit instructions (Shanghai Yaji Biotech Co., Ltd., China). TBA was purchased from Beijing Biolab Technology Co., Ltd., China. The OD value of the test sample supernatant was determined at $532 \mathrm{~nm}$. 


\subsection{Reverse transcription-polymerase chain reaction (RT-PCR) detection of PI3K and mTOR mRNA levels}

RHiN cells were cultured to logarithmic phase after appropriate treatments. Following cell collection, total RNA was extracted by TRIzol reagent (obtained from Shanghai Yeasen Biotechnology Co., Ltd., China). A nanodrop spectrophotometer (Thermo Fisher Scientific Inc., USA) was used for verifying the quality of RNA samples. Complementary DNA (cDNA) libraries were synthesized by RNA reverse transcription kit (Shanghai Yeasen Biotechnology Co., Ltd., China). Reaction conditions were set at $42^{\circ} \mathrm{C}$ for $15 \mathrm{~min}$ and $80^{\circ} \mathrm{C}$ for 5 min. The product was stored at $4^{\circ} \mathrm{C}$. Glyceraldehyde3-phosphate dehydrogenase (GAPDH) was used as the reference gene for the purpose of this study. Primer 5.0 (Suzhou Genewiz Biology Company, China) was used to design forward and reverse amplification primers. For PI3K, the upstream primer sequence was 5'-CACTGGCATCCTCACTCAC-3' and downstream primer sequence was 5'-CTGACTGACTCACTGC-3'. For mTOR, the upstream primer sequence was 5'-CGTCAACCATCCATGTAC-3' and the downstream primer was 5'-CGTACTGCATGCACTCATGCC-3'. For GAPDH, the upstream primer was 5'-TCACTGCACTGCA-CTGCAC-3', and the downstream primer was 5'-CGCTGACTGACGTCAC-3'. Reaction conditions were initiated at $95^{\circ} \mathrm{C}$ for $30 \mathrm{~s}, 95^{\circ} \mathrm{C}$ for $5 \mathrm{~s}, 62^{\circ} \mathrm{C}$ for $20 \mathrm{~s}, 72^{\circ} \mathrm{C}$ for $30 \mathrm{~s}$, and the reaction was run for 32 cycles. The $2^{-\Delta c t}$ was used to calculate relative expression levels of target genes.

\subsection{Western blot analysis of PI3K and mTOR protein expression}

RHiN cells in the logarithmic phase were collected. RIPA lysis buffer was used to treat cells for $15 \mathrm{~min}$ prior to total protein extraction. Protein concentration was determined using the bicinchoninic acid assay (BCA assay). The protein sample solution and $5 \times$ protein loading buffer (obtained from Beyotime Biotechnology Company, China) were mixed. After boiling for $6 \mathrm{~min}$, samples were cooled to room temperature. Denatured protein $(15 \mu \mathrm{g})$ was added to sample wells of $10 \%$ of sodium dodecyl sulfate polyacrylamide gel electrophoresis (SDS-PAGE) gels, and proteins were electrophoresed. Following electrophoresis, total proteins were transferred to polyvinylidene fluoride (PVDF) membrane (Shanghai Yeasen Biotechnology Co., Ltd., China) by wet-transfer method. Confining liquid with $5 \%$ skim milk powder was used to block the membrane with proteins for
$1 \mathrm{~h}$. Rabbit anti-rat PI3K and mTOR protein monoclonal antibodies (dilution ratio of 1:800 and 1:1,400 respectively) (Beijing Bioss Biotech Co., Ltd., China) were added, placed at $4^{\circ} \mathrm{C}$ for incubation overnight. After tris buffered saline with tween (TBST) washing the membrane, HRP-labeled sheep anti-rabbit IgG secondary antibody (dilution ratio of 1:2,000) (Beijing Bioss Biotech Co., Ltd., China) was incubated with the membrane for $1 \mathrm{~h}$. Electrogenerated chemiluminescence (ECL) was used to detect labelled proteins on the membrane. Exposure and photography were performed, followed by film analysis. Image J software was used to measure the gray values of selected protein stripes.

\subsection{Protective effects of miR-182 on cerebral ischemic oxidative stress model rats}

Thirty 10-week-old clean-grade male Wistar rats (Beijing Vital River Laboratory Animal Technology Co., Ltd., China) were purchased with a body weight of 250-300 $\mathrm{g}$, feeding temperature of 22-25 degrees and relative humidity of 45\%-55\% (license number: SCXK2004-2005). Rats were divided into blank control group, model control group and miR-182 group, with 10 rats in each group. The rats of miR-182 group were injected with miR-182 (15 ng/d) via tail vein injection continuously for 7 days, and the rats of the other two groups were given free diet. On the 8th day, a model of global cerebral ischemia was established by modified Longa method in model control group and miR-182 group. After 90 min of ischemia in rats, reperfusion was performed for $24 \mathrm{~h}$. After anesthesia, blood was collected from the abdominal aorta and rats were killed by cervical dislocation. Hippocampal sections were collected and hippocampal neuron cells were isolated to detect cell viability, apoptosis levels, serum SOD and MDA levels. Hippocampal sections were prepared following paraffinembedded treatment. Sections were cut to a thickness of $5 \mu \mathrm{m}$, and then placed in a $60^{\circ} \mathrm{C}$ incubator for $2 \mathrm{~h}$. Xylene (Shanghai Yeasen Biotechnology Co., Ltd., China) was used for dewaxing and hydration, then incubated for $10 \mathrm{~min}$ in a 3\% solution of $\mathrm{H}_{2} \mathrm{O}_{2}$. PBS was used to rinse the samples, and pH 6.0 citrate buffer (Shanghai Yeasen Biotechnology Co., Ltd., China) was added to the tissue. Tissue samples were boiled for $15 \mathrm{~min}$. After cooling, 5\% goat serum (Sangon Biotech (Shanghai) Co., Ltd., China) was dropped and then closed for $1 \mathrm{~h}$. After rinsing with PBS, rabbit antirat PI3K and mTOR protein primary antibodies (Beijing Bioss Biotech Co., Ltd., China) were added to the tissue, and incubated at room temperature for $2 \mathrm{~h}$. PBS washes were carried out to remove primary antibody from the samples, and sheep anti-rabbit IgG secondary antibody 
(Beijing Bioss Biotech Co., Ltd., China) was added for incubation at room temperature for $1 \mathrm{~h}$. Diaminobenzidine (DAB) (Sangon Biotech (Shanghai) Co., Ltd., China) was added for straining following the secondary antibody washes. Tissue samples were re-strained, dehydrated and hyalinized, followed by application of a neutral gum seal before being placed under the microscope (Olympus Corporation, Japan) for observation and documentation.

Ethical approval: The research related to animal use has complied with all the relevant national regulations and institutional policies for the care and use of animals.

\subsection{Statistical analysis}

SPSS19.0 software was used to analyze data. The measurement data were expressed as mean \pm standard deviation ( $\bar{x} \pm$ sd). One-way ANOVA was used for comparison of multiple samples. Dunnett's post hoc $t$ test was used for comparison between groups. $\mathrm{P}<0.05$ indicated statistically significant differences.

\section{Results}

\subsection{The effect of miR-182 transfection on survival rates and apoptosis of RHiN cells induced by $\mathrm{H}_{2} \mathrm{O}_{2}$}

The survival rates of RHiN cells in miR-182 group and mTOR activator group were $117.56 \pm 6.75 \%$ and $116.48 \pm 7.28 \%$, significantly higher than blank control group (both
$\mathrm{P}<0.05)$. The survival rates of RHiN cells in $\mathrm{H}_{2} \mathrm{O}_{2}$ group and mTOR inhibitor group were $57.62 \pm 3.94 \%$ and $64.39 \pm 4.95 \%$, significantly lower than that in blank control group (both $\mathrm{P}<0.05)$. The survival rates of RHiN cells in $\mathrm{H}_{2} \mathrm{O}_{2}+$ miR-182 group and $\mathrm{H}_{2} \mathrm{O}_{2}+\mathrm{mTOR}$ group were $73.64 \pm 5.37 \%$ and $78.13 \pm 6.51 \%$ respectively, significantly higher than that observed in $\mathrm{H}_{2} \mathrm{O}_{2}$ group (both $\mathrm{P}<0.05$ ). Flow cytometry analysis indicated that the apoptosis rate of RHiN cells in $\mathrm{H}_{2} \mathrm{O}_{2}$ group was $22.36 \pm 3.15 \%$, significantly higher than blank control group $(\mathrm{P}<0.05)$. The apoptosis rate of RHiN cells in miR-182 group was $4.27 \pm 1.12 \%$, significantly lower than blank control group $(\mathrm{P}<0.05)$. The apoptosis rates of RHiN cells in $\mathrm{H}_{2} \mathrm{O}_{2}+$ miR-182 group and $\mathrm{H}_{2} \mathrm{O}_{2}+$ mTOR group were $8.14 \pm 2.18 \%$ and $9.32 \pm 2.43 \%$ respectively, significantly lower than that in $\mathrm{H}_{2} \mathrm{O}_{2}$ group (both $\mathrm{P}<0.05$ ), as shown in Figure 1.

\subsection{Effect of miR-182 transfection on SOD and MDA in RHiN cells}

MDA levels in RHiN cells from miR-182 group and mTOR activator group were $0.34 \pm 0.03 \mu \mathrm{mol} / \mathrm{mL}$ and $0.31 \pm 0.05$ $\mu \mathrm{mol} / \mathrm{mL}$, significantly lower than blank control group (both $\mathrm{P}<0.05$ ). MDA levels in RHiN cells from $\mathrm{H}_{2} \mathrm{O}_{2}$ group and mTOR inhibitor group were $0.75 \pm 0.08 \mu \mathrm{mol} / \mathrm{mL}$ and $0.79 \pm 0.07 \mu \mathrm{mol} / \mathrm{mL}$, significantly higher than blank control group (both $\mathrm{P}<0.05$ ). MDA levels in RHiN cells from $\mathrm{H}_{2} \mathrm{O}_{2}+$ miR-182 group and $\mathrm{H}_{2} \mathrm{O}_{2}+$ mTOR group were $0.47 \pm 0.05 \mu \mathrm{mol} / \mathrm{mL}$ and $0.43 \pm 0.04 \mu \mathrm{mol} / \mathrm{mL}$, significantly lower than $\mathrm{H}_{2} \mathrm{O}_{2}$ group (both $\mathrm{P}<0.05$ ). SOD levels in RHiN cells from miR-182 group and mTOR activator group were $17.75 \pm 0.49 \mathrm{U} / \mathrm{mL}$ and $18.14 \pm 0.74 \mathrm{U} / \mathrm{mL}$, significantly higher than blank control group (both
A

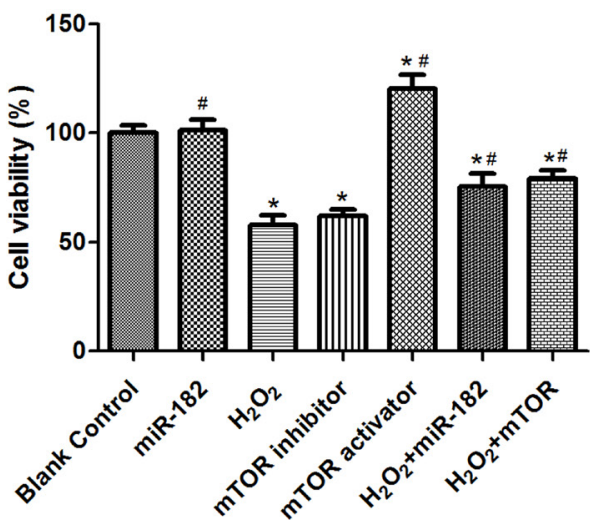

B

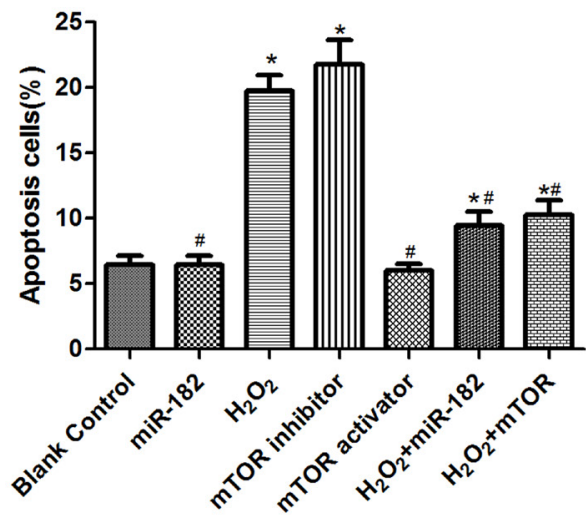

Figure 1. The survival rate and apoptosis of RhiN in each group was determined. A: The survival rates of RHiN in each group. B: The apoptosis rates of $\mathrm{RHiN}$ in each group. Compared with balnk control group, ${ }^{*} \mathrm{P}<0.05$; compared with $\mathrm{H}_{2} \mathrm{O}_{2}$ group, ${ }^{\#} \mathrm{P}<0.05$. RhiN: rat hippocampal neurons. 
$\mathrm{P}<0.05)$. SOD levels in RHiN cells from $\mathrm{H}_{2} \mathrm{O}_{2}$ group and mTOR inhibitor group were $5.34 \pm 0.12 \mathrm{U} / \mathrm{mL}$ and $6.04 \pm 0.16 \mathrm{U} / \mathrm{mL}$, significantly lower than blank control group (both $\mathrm{P}<0.05$ ). SOD levels in RHiN cells from $\mathrm{H}_{2} \mathrm{O}_{2}+$ miR-182 group and $\mathrm{H}_{2} \mathrm{O}_{2}+$ mTOR group were $11.42 \pm 0.42$ $\mathrm{U} / \mathrm{mL}$ and $12.37 \pm 0.62 \mathrm{U} / \mathrm{mL}$, significantly higher than those in $\mathrm{H}_{2} \mathrm{O}_{2}$ group (both $\mathrm{P}<0.05$ ), as illustrated in Figure 2.

\subsection{Effect of miR-182 transfection on mTOR and PI3K mRNA levels in RHiN cells}

RT-PCR experiments showed that the mTOR mRNA level in RHiN cells from mTOR activator group was $1.17 \pm 0.19$, higher than blank control group $(\mathrm{P}<0.05)$. The mTOR mRNA levels in RHiN cells from $\mathrm{H}_{2} \mathrm{O}_{2}$ group and mTOR inhibitor group were $0.64 \pm 0.08$ and $0.43 \pm 0.06$, lower than blank control group (both $\mathrm{P}<0.05$ ). The mTOR mRNA levels in RHiN cells from $\mathrm{H}_{2} \mathrm{O}_{2}+$ miR-182 group and $\mathrm{H}_{2} \mathrm{O}_{2}+$ mTOR group were $0.82 \pm 0.13$ and $0.85+0.14$, significantly higher than $\mathrm{H}_{2} \mathrm{O}_{2}$ group (both $\mathrm{P}<0.05$ ). The PI3K mRNA level in miR-182 group was $1.13 \pm 0.03$, significantly higher than $\mathrm{H}_{2} \mathrm{O}_{2}$ group $(\mathrm{P}<0.05)$. The PI3K mRNA level in $\mathrm{H}_{2} \mathrm{O}_{2}$ group was $0.48 \pm 0.04$, significantly lower than blank control group $(\mathrm{P}<0.05)$. The PI3K mRNA levels in $\mathrm{H}_{2} \mathrm{O}_{2}+$ miR-182 group and $\mathrm{H}_{2} \mathrm{O}_{2}+\mathrm{mTOR}$ group were $0.87 \pm 0.06$ and $0.89 \pm 0.09$, which were significantly higher than those in $\mathrm{H}_{2} \mathrm{O}_{2}$ group (both $\mathrm{P}<0.05$ ). Comparisons of the mRNA expression levels are shown in Figure 3.
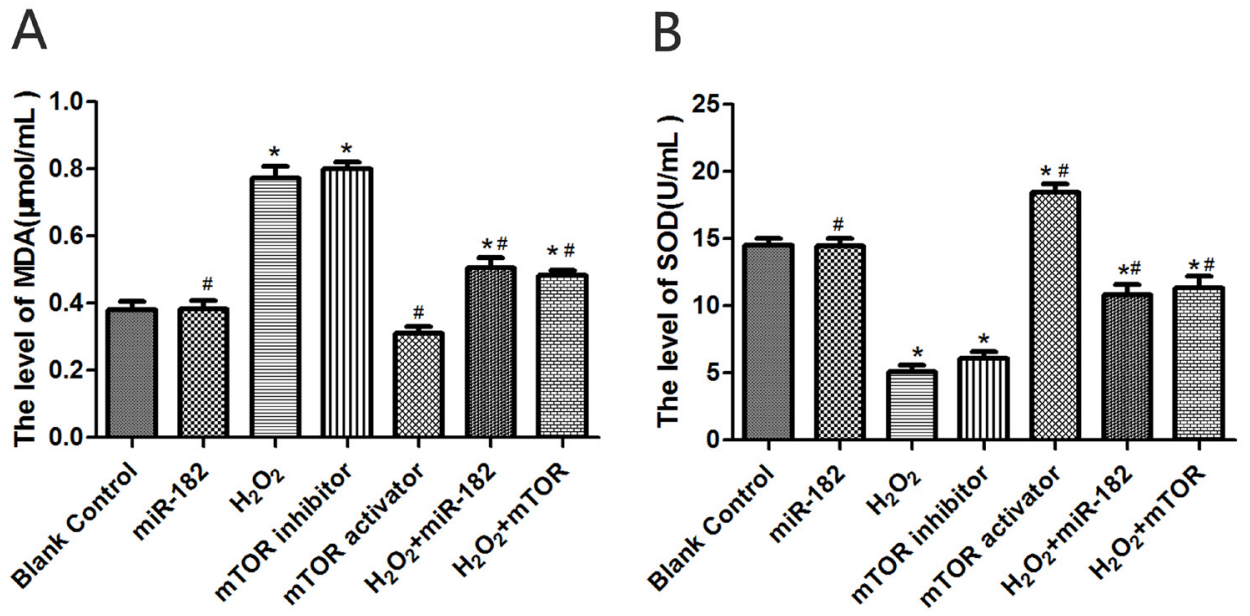

Figure 2. MDA levels and SOD levels in RhiN. A: MDA levels in RHiN of each group. B: SOD levels in RHiN of each group. Compared with balnk control group, ${ }^{*} \mathrm{P}<0.05$; compared with $\mathrm{H}_{2} \mathrm{O}_{2}$ group, ${ }^{\mathrm{P}} \mathrm{P}<0.05$. MDA: malondialdehyde; SOD: superoxide dismutase; RhiN: rat hippocampal neurons.
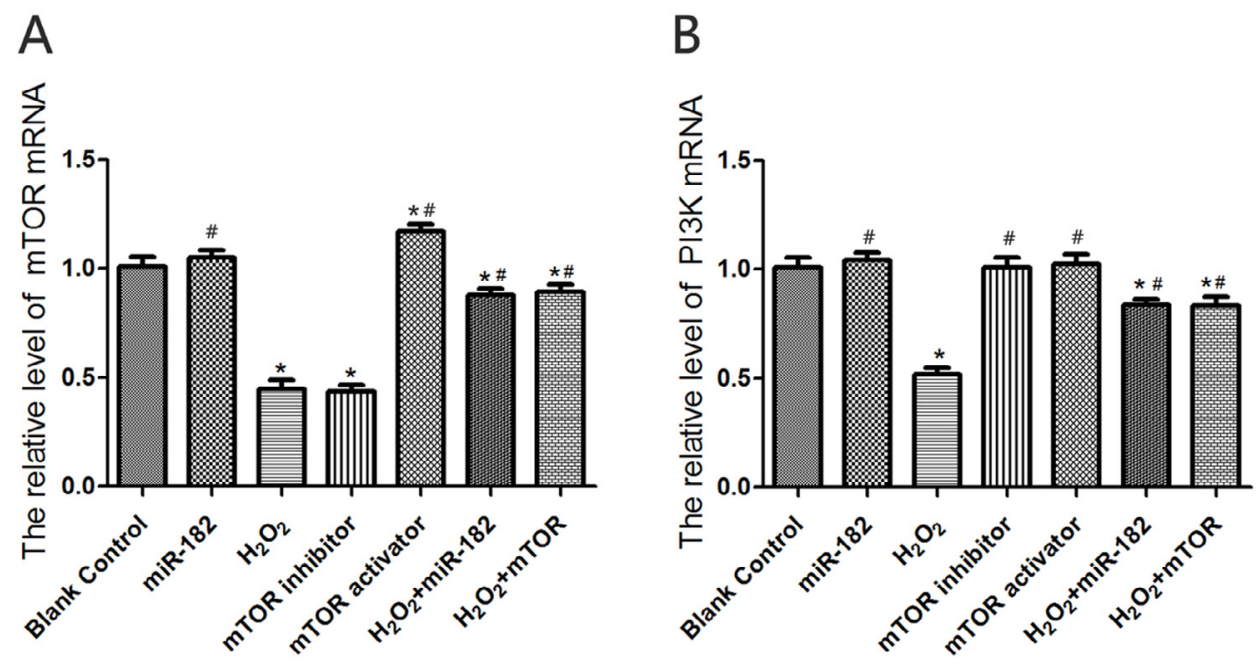

Figure 3. $m$ TOR and PI3K mRNA levels in RHiN. A: The mTOR mRNA levels in hippocampal neurons in each group. B: The PI3K mRNA levels in hippocampal neurons in each group. Compared with balnk control group, ${ }^{*} \mathrm{P}<0.05$; compared with $\mathrm{H}_{2} \mathrm{O}_{2}$ group, ${ }^{*} \mathrm{P}<0.05$. mTOR: mammalian target of rapamycin; PI3K: phosphatidylinositol 3-kinase; RhiN: rat hippocampal neurons. 


\subsection{The effect of miR-182 on mTOR and PI3K protein levels in RHiN cells}

Western Blot evaluation of protein expressions determined that the expression level of mTOR protein in mTOR activator group was $0.71 \pm 0.18$, higher than blank control group $(\mathrm{P}<0.05)$. The expression levels of mTOR protein in $\mathrm{H}_{2} \mathrm{O}_{2}$ group and mTOR inhibitor group were $0.18 \pm 0.03$ and $0.23 \pm 0.04$, lower than blank control group (both $\mathrm{P}<0.05$ ). The expression levels of mTOR protein in $\mathrm{H}_{2} \mathrm{O}_{2}+$ miR-182 group and $\mathrm{H}_{2} \mathrm{O}_{2}+$ mTOR group were $0.45 \pm 0.06$ and $0.42 \pm 0.04$, significantly higher than $\mathrm{H}_{2} \mathrm{O}_{2}$ group (both $\mathrm{P}<0.05$ ). The expression level of PI3K protein in miR-182 group was $0.57 \pm 0.08$, significantly higher than $\mathrm{H}_{2} \mathrm{O}_{2}$ group $(\mathrm{P}<0.05)$. The expression level of $\mathrm{PI} 3 \mathrm{~K}$ protein in $\mathrm{H}_{2} \mathrm{O}_{2}$ group was $0.23 \pm 0.06$, significantly lower than blank control group $(\mathrm{P}<0.05)$. The expression levels of PI3K protein in $\mathrm{H}_{2} \mathrm{O}_{2}+$ miR-182 group and $\mathrm{H}_{2} \mathrm{O}_{2}+$ mTOR group were $0.36 \pm 0.04$ and $0.38 \pm 0.05$, significantly higher than $\mathrm{H}_{2} \mathrm{O}_{2}$ group (both $\mathrm{P}<0.05$ ). Comparisons of the protein expression levels are shown in Figure 4 and Figure 5.

\subsection{Protective effects of miR-182 on cerebral ischemic oxidative stress model rats}

The survival rate and SOD level of rat hippocampal neuronal cells in miR-182 group were $62.15 \pm 4.28 \%$ and $11.36 \pm 2.41 \mathrm{U} / \mathrm{mL}$ respectively, significantly higher than model control group (both $\mathrm{P}<0.05$ ). The apoptosis rate and MDA level of rat hippocampal neuronal cells in miR-182 group were $13.12 \pm 2.16 \%$ and $0.41 \pm 0.05 \mu \mathrm{mol} /$ $\mathrm{mL}$, significantly lower than model control group (both
$\mathrm{P}<0.05)$. Immunohistochemical staining showed that the intensities of mTOR and PI3K in model group were $124.57 \pm 18.43$ and $87.26 \pm 18.43$ respectively, significantly lower than blank control group $(\mathrm{P}<0.05)$. Positive staining intensities of mTOR and PI3K were $141.25 \pm 16.52$ and $124.18 \pm 19.49$ respectively in miR-182 group, which were significantly higher than that in model blank group (both $\mathrm{P}<0.05$ ), as shown in Figure 6, Figure 7 and Figure 8.

\section{Discussion}

The hippocampus is central to information storage and memory functions in the brain. Oxidative stress injury to RHiN cells can lead to neurological deficits in the brain. Furthermore, oxidative injuries may cause various

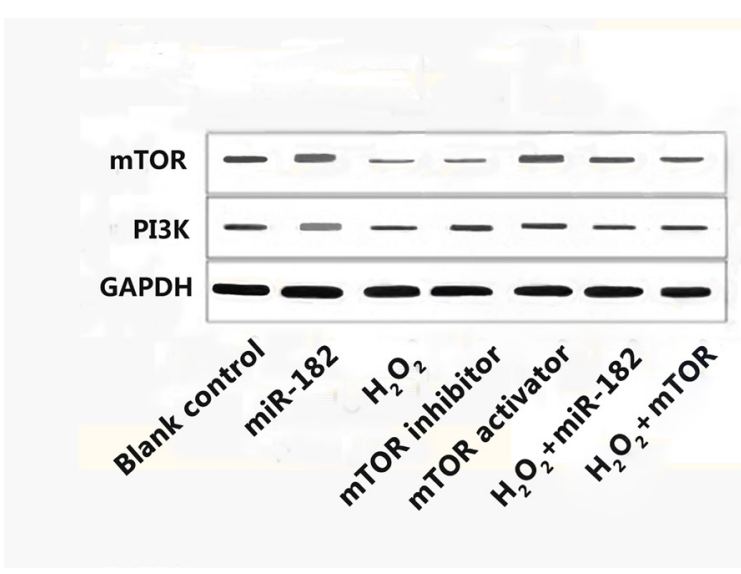

Figure 4. Electrophoretogram of mTOR and PI3K in RHiN. mTOR: mammalian target of rapamycin; PI3K: phosphatidylinositol 3-kinase; GAPDH: Glyceraldehyde-3-phosphate dehydrogenase; RhiN: rat hippocampal neurons.
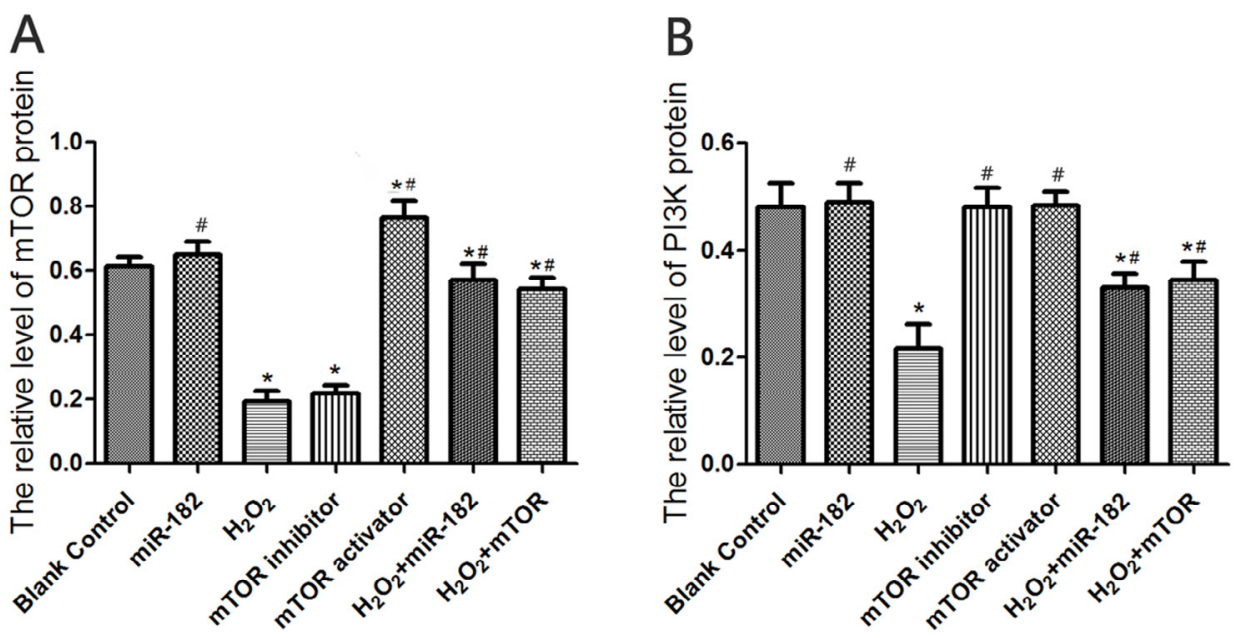

Figure 5. $\mathrm{mTOR}$ and $\mathrm{PI3K}$ protein levels in RHiN cells. A: Values of expression levels of mTOR protein in each group. B: Values of expression levels of $\mathrm{PI} 3 \mathrm{~K}$ protein in each group. Compared with blank control group, ${ }^{*} \mathrm{P}<0.05$; compared with $\mathrm{H}_{2} \mathrm{O}_{2}$ group, ${ }^{*} \mathrm{P}<0.05$. mTOR: mammalian target of rapamycin; PI3K: phosphatidylinositol 3-kinase; RhiN: rat hippocampal neurons. 
neurodegenerative diseases such as Alzheimer's disease and Parkinson's disease [8]. Atalay et al. have found that the memory and neural activity of rats decreases significantly after oxidative stress in RHiN cells [9]. The apoptosis rate of neurons increased after the increase in oxidative stress in the hippocampus, and the rats appeared to suffer from cognitive impairment [10]. When oxygen free radicals (such as ROS and NOS) cannot be effectively removed from cells, active constituents such as DNA, protein and other macromolecules are attacked. Further, the potential of the mitochondrial membrane is changed, and neuronal apoptosis is induced as a result [11, 12]. Therefore, effective enhancement of the antioxidant capacity of neurons is of great value in reducing oxidative stress injury.
In this study, cell viability of RHiN cells in the $\mathrm{H}_{2} \mathrm{O}_{2}+$ miR-182 group was increased after miR-182 transfection, as demonstrated by effective reduction of cellular apoptosis. Apoptosis results indicate that miR-182 is involved in regulating the oxidative stress response of rat neurons, which effectively reduces the damage incurred from oxidative stress. SOD is an enzyme that can effectively remove free radicals in vivo, thereby influencing cellular antioxidant levels. When the levels of SOD are reduced, there are excess free radicals without an effective removal system. Oxidative damage of brain neurons from this excess of radicals has been reported $[13,14]$. MDA is the end-product of cells attacked by free radicals. It is cytotoxic and can exacerbate damage to cellular functions [15-17]. Zhang et al. reported that SOD
A

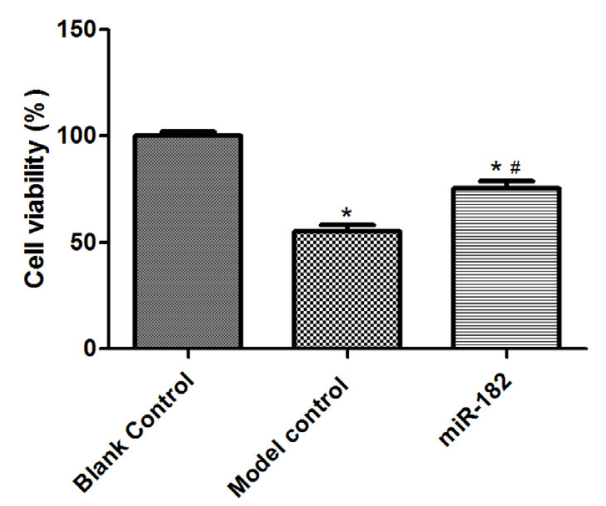

B

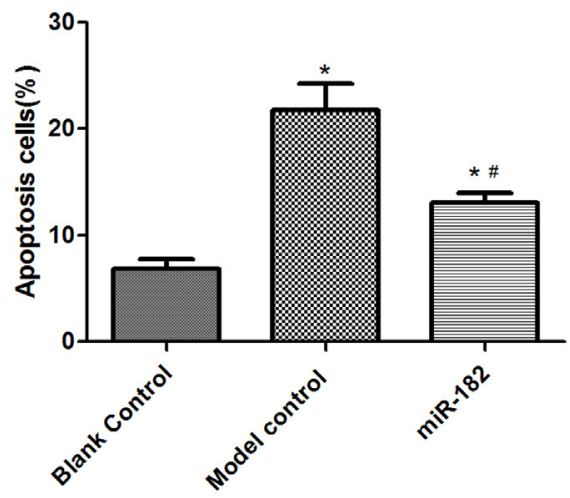

Figure 6. The survival rate and apoptosis of rat hippocampal neuronal cells in each group. A: The survival rate of rat hippocampal neuronal cells. B: The apoptosis of rat hippocampal neuronal cells. Compared with blank control group, ${ }^{*} \mathrm{P}<0.05$; compared with $\mathrm{H}_{2} \mathrm{O}_{2}$ group, ${ }^{\#} \mathrm{P}<0.05$.

A

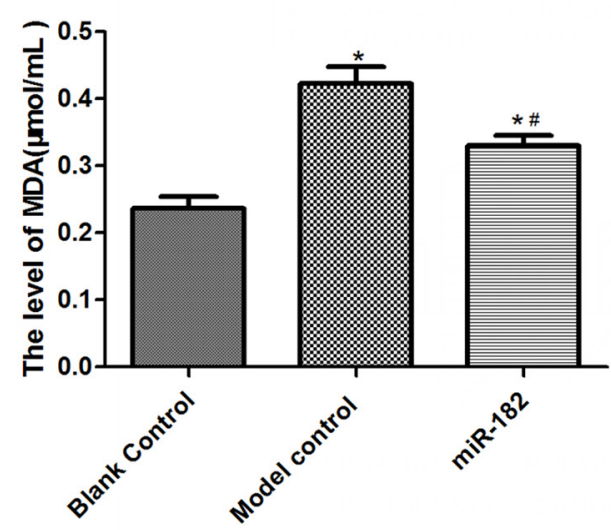

B

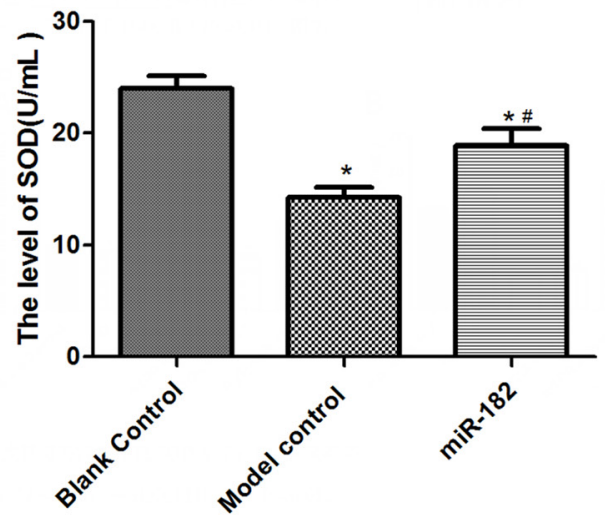

Figure 7. MDA levels and SOD levels in serum of rats. A: MDA levels in rat hippocampal neuronal cells. B: SOD levels in rat hippocampal neuronal cells. Compared with balnk control group, ${ }^{*} \mathrm{P}<0.05$; compared with model control group, ${ }^{\#} \mathrm{P}<0.05$. MDA: malondialdehyde; SOD: superoxide dismutase. 
levels of retinal ganglion cells are significantly reduced after oxidative stress damage, and the reduction is associated with excitatory transmission dysfunction of the neurons [18]. In this experiment, SOD levels in treated RHiN cells were decreased after $\mathrm{H}_{2} \mathrm{O}_{2}$ treatment and MDA levels were increased, suggesting higher levels of oxidative stress compared to controls. After transfection of miR-182 in rat neurons, the levels of SOD increased and MDA levels decreased. This affect indicates that miR-182 transfection alleviates oxidative stress in neuronal cells, which is consistent with previous studies [19].

The PI3K-AKT-mTOR signaling pathway plays an important role in regulating cell growth, apoptosis, autophagy and cellular antioxidant capacity. PI3K is a key protein in the signaling pathway, as it can be phosphorylated to form phospholipid creatine phosphate, thereby prompting the phosphorylation of AKT kinase [20]. mTOR is a serine/threonine protein kinase that regulates the activity of ribosomal S6 protein kinase
(S6K) and eukaryotic promoter 4E binding protein 1 (4E-BP1), which further act to regulate downstream gene transcription and protein synthesis [21-23]. Jiao et al. have found that increased levels of mTOR and PI3K proteins in cardiac myocytes can increase antioxidant levels in the cells. The higher levels are associated with alleviating free radical damage, thus enhancing the cells' viability under adverse conditions [1]. Huang $W$ et al. have also found that the myocytes are sensitive to oxidative stress and prone to apoptosis after treatment with an mTOR-specific antagonist [24]. The results of this study demonstrate the intensified oxidative stress reaction of RHiN cells after treatment with $\mathrm{H}_{2} \mathrm{O}_{2}$. The expression levels of MTOR and PI3K proteins were decreased as a result of the peroxide challenge. Transfection of miR-182 increased the expression of mTOR and PI3K proteins in rat neurons and activated the PI3K-AKT-mTOR signaling pathway, with its concomitant positive effects on relieving neuronal oxidative stress. Our results suggest that miR-182 increases

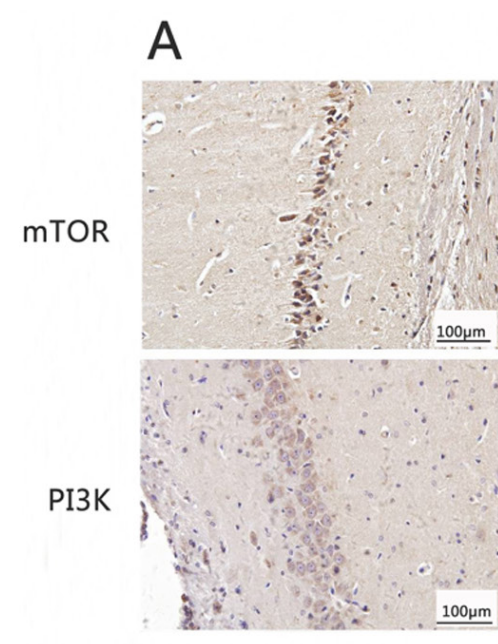

D

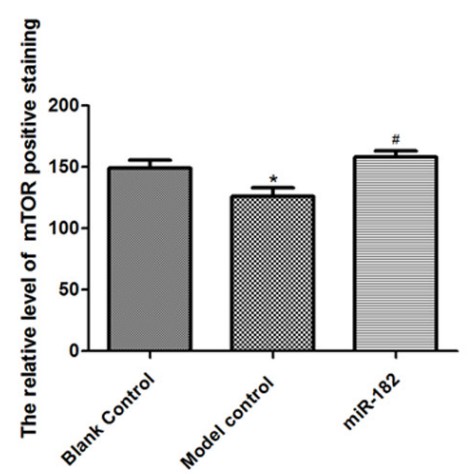

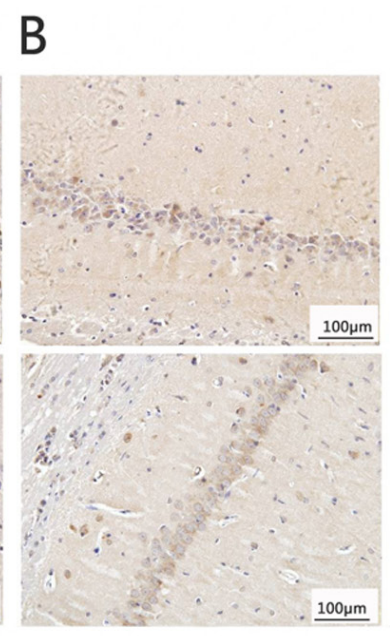

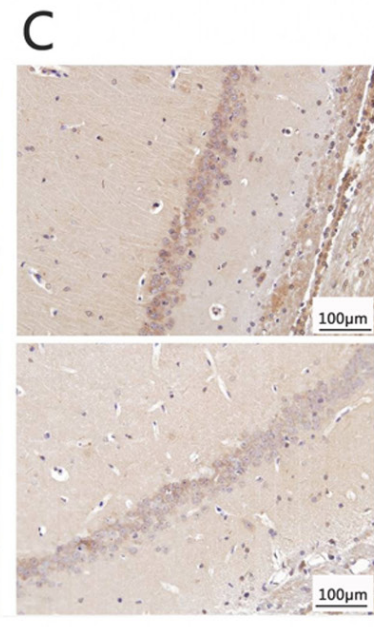

E

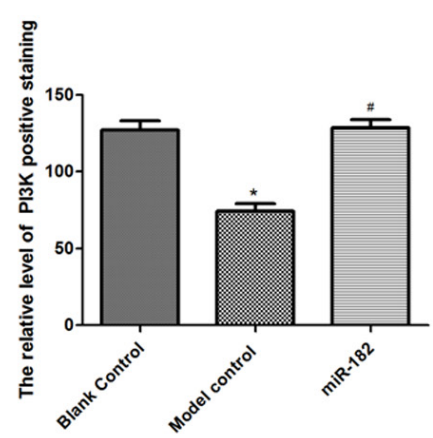

Figure 8. Immunohistochemistry staining of rat hippocampus tissue (200X). A: mTOR and PI3K protein staining in rat hippocampus tissue in blank control group. B: $\mathrm{mTOR}$ and PI3K protein staining in rat hippocampus tissue in model control group. C: $\mathrm{mTOR}$ and PI3K protein staining in rat hippocampus tissue in miR-182 group. D: The positive staining intensity of mTOR protein in rat hippocampus tissue of each group. E: The positive staining intensity of $\mathrm{PI} 3 \mathrm{~K}$ in rat hippocampus tissue of each group. Compared with blank control group, ${ }^{*} \mathrm{P}<0.05$; compared with model control group, ${ }^{\mathrm{P}<0.05}$. mTOR: mammalian target of rapamycin; $\mathrm{PI} 3 \mathrm{~K}$ : phosphatidylinositol 3-kinase. 
the antioxidant capacity of neuronal cells. At the same time, our study showed that miR-182 could alleviate the damage of oxidative stress on rat hippocampal neuronal cells in cerebral ischemic oxidative stress model rats. The mechanism of action of miR-182's influence on the expression of mTOR and PI3K in rat neurons is beyond the scope of this study, however our initial indications warrant further investigations of related mechanisms.

In conclusion, miR-182 can effectively alleviate oxidative damage to rat hippocampal neurons induced by $\mathrm{H}_{2} \mathrm{O}_{2}$. The mechanism of miR-182's efficacy involves activation of the PI3K-AKT-mTOR signaling pathway as a means for alleviating the damage from free radicals within the neuronal cells.

Acknowledgement: This work was supported by Open Platform Project of Hunan Education Department (15K106).

Conflict of interest: Authors state no conflict of interest.

\section{References}

[1] Jiao W, Wang Y, Kong L, Ouyang T, Meng Q, Fu Q, et al. CART peptide activates the Nrf2/HO-1 antioxidant pathway and protects hippocampal neurons in a rat model of Alzheimer's disease. Biochem Biophys Res Commun. 2018;501(4):1016-22.

[2] Lu XT, Ma Y, Zhang HJ, Jin MQ, Tang JH. Enantioselective apoptosis and oxidative damage induced by individual isomers of profenofos in primary hippocampal neurons. J Environ Sci Health B. 2017;52(7):505-15.

[3] Heinemann SD, Posimo JM, Mason DM, Hutchison DF, Leak RK. Synergistic stress exacerbation in hippocampal neurons: Evidence favoring the dual-hit hypothesis of neurodegeneration. Hippocampus. 2016;26(8):980-94.

[4] Gong HY, Zheng F, Zhang C, Chen XY, Liu JJ, Yue XQ. Propofol protects hippocampal neurons from apoptosis in ischemic brain injury by increasing GLT-1 expression and inhibiting the activation of NMDAR via the JNK/Akt signaling pathway. Int J Mol Med. 2016;38(3):943-50.

[5] Yuliani S, Widyarini S, Mustofa, Partadiredja G. Turmeric extract inhibits apoptosis of hippocampal neurons of trimethyltinexposed rats. Bratisl Lek Listy. 2017;118(3):142-8.

[6] Wang CP, Shi YW, Tang M, Zhang XC, Gu Y, Liang XM, et al. Isoquercetin Ameliorates Cerebral Impairment in Focal Ischemia Through Anti-Oxidative, Anti-Inflammatory, and Anti-Apoptotic Effects in Primary Culture of Rat Hippocampal Neurons and Hippocampal CA1 Region of Rats. Mol Neurobiol. 2017;54(3):2126-42.

[7] Kho AR, Choi BY, Kim JH, Lee SH, Hong DK, Lee SH, et al. Prevention of hypoglycemia-induced hippocampal neuronal death by N-acetyl-L-cysteine (NAC). Amino Acids. 2017;49(2):367-78.

[8] Singh AK, Kashyap MP, Tripathi VK, Singh S, Garg G, Rizvi SI. Neuroprotection Through Rapamycin-Induced Activation of Autophagy and PI3K/Akt1/mTOR/CREB Signaling Against Amyloid- $\beta$-Induced Oxidative Stress, Synaptic/Neurotransmission Dysfunction, and Neurodegeneration in Adult Rats. Mol Neurobiol. 2017;54(8):5815-28.

[9] Atalay T, Gulsen I, Colcimen N, Alp HH, Sosuncu E, Alaca I. Resveratrol treatment prevents hippocamal neurodegeneration in a rodent model of traumatic brain injury. Turkish Neurosurgery. 2016.

[10] Karkhah A, Ataee R, Ataie A. Morphine pre- and post-conditioning exacerbates apoptosis in rat hippocampus cells in a model of homocysteine-induced oxidative stress. Biomed Rep. 2017;7(4):309-13.

[11] Sheng L, Ze Y, Wang L, Yu X, Hong J, Zhao X, et al. Mechanisms of $\mathrm{TiO}_{2}$ nanoparticle-induced neuronal apoptosis in rat primary cultured hippocampal neurons. J Biomed Mater Res A. 2015;103(3):1141-9.

[12] Ma NT, Zhou R, Chang RY, Hao YJ, Ma L, Jin SJ. Protective effects of aloperine on neonatal rat primary cultured hippocampal neurons injured by oxygen-glucose deprivation and reperfusion. J Nat Med. 2015;69(4):575-83.

[13] Agarwal S, Tiwari SK, Seth B, Yadav A, Singh A, Mudawal A, et al. Activation of Autophagic Flux against Xenoestrogen Bisphenol-A-induced Hippocampal Neurodegeneration via AMP kinase (AMPK)/Mammalian Target of Rapamycin (mTOR) Pathways. J Biol Chem. 2015;290(34):21163-84.

[14] De-Paula VJ, Gattaz WF, Forlenza OV. Long-term lithium treatment increases intracellular and extracellular brainderived neurotrophic factor (BDNF) in cortical and hippocampal neurons at subtherapeutic concentrations. Bipolar Disord. 2016;18(8):692-5.

[15] Zhao YN, Wang HY, Jian-Min LI, Chen BY, Xia G, Zhang PP. Hippocampal mitogen-activated protein kinase activation is associated with intermittent hypoxia in a rat model of obstructive sleep apnea syndrome. Mol Med Rep. 2016;13(1):137-45.

[16] Angamo EA, Roesner J, Liotta A, Kovacs R, Heinemann $U$. A neuronal lactate uptake inhibitor slows recovery of extracellular ion concentration changes in the hippocampal CA3 region by affecting energy metabolism. J Neurophysiol. 2016;116(5):2420-2430.

[17] Godoy JA, Lindsay CB, Quintanilla RA, Carvajal FJ, Cerpa W, Inestrosa NC. Quercetin Exerts Differential Neuroprotective Effects Against $\mathrm{H}_{2} \mathrm{O}_{2}$ and $A \beta$ Aggregates in Hippocampal Neurons: the Role of Mitochondria. Mol Neurobiol. 2016;54(9):7116-28.

[18] Zhang Y, Gao L, Cheng Z, Cai J, Niu Y, Meng W, et al. Kukoamine A Prevents Radiation-Induced Neuroinflammation and Preserves Hippocampal Neurogenesis in Rats by Inhibiting Activation of NF-KB and AP-1. Neurotox Res. 2017;31(2):259-68.

[19] Mohibbullah M, Hannan MA, Choi JY, Bhuiyan MM, Hong YK, Choi JS, et al. The Edible Marine Alga Gracilariopsis chorda Alleviates Hypoxia/Reoxygenation-Induced Oxidative Stress in Cultured Hippocampal Neurons. J Med Food. 2015;18(9):960-71.

[20] Patel B, Das SK, Das S, Das L, Patri M. Neonatal exposure to benzo[a]pyrene induces oxidative stress causing altered hippocampal cytomorphometry and behavior during early adolescence period of male Wistar rats. International Journal of Developmental Neuroscience. 2016;50(50):7-15.

[21] Guo J, Yang CX, Yang JJ, Yao Y. Glycyrrhizic acid ameliorates cognitive impairment on vascular dementia model rats 
associated with oxidative damage and inhibition of voltagegated sodium channels. CNS Neurol Disord Drug Targets. 2016;15(8):1001-8.

[22] Yazğan Y, Nazıroğlu M. Ovariectomy-Induced Mitochondrial Oxidative Stress, Apoptosis, and Calcium Ion Influx Through TRPA1, TRPM2, and TRPV1 Are Prevented by $17 \beta$-Estradiol, Tamoxifen, and Raloxifene in the Hippocampus and Dorsal Root Ganglion of Rats. Mol Neurobiol. 2017;54(10):7620-38.
[23] Tomasini MC, Borelli AC, Beggiato S, Tanganelli S, Loche A, Cacciaglia R, et al. GET73 Prevents Ethanol-Induced Neurotoxicity in Primary Cultures of Rat Hippocampal Neurons. Alcohol Alcohol. 2016;51(2):128-35.

[24] Huang W, Meng F, Jie C, Liu X, Jie Z, Min L. Neuroprotective Role of Exogenous Brain-Derived Neurotrophic Factor in Hypoxia-Hypoglycemia-Induced Hippocampal Neuron Injury via Regulating Trkb/MiR134 Signaling. J Mol Neurosci. 2017;62(1):35-42. 